\title{
Perfil das infecções fúngicas em pacientes transplantados renais
}

\author{
Profile of fungal infectios in renal transplantes patients \\ 1*Fontenele, Andréa Martins Melo; ${ }^{2}$ Rodrigues Hemmerson Gomes ${ }^{3}$ Froes Soraya María da \\ Rocha; ${ }^{3}$ Carvahal Rafael Santos; ${ }^{4}$ Zaror Luis \\ ${ }^{11}$ Farmacêutica responsável da Unidade de Transplante Renal do Hospital Universitário da
Universidade Federal do Maranhão - HUUFMA.
${ }^{2}$ Farmacêutico residente do Hospital Universitário da Universidade Federal do Maranhão - HUUFMA.
${ }^{3}$ Unidade de Transplante HUUFMA.
${ }^{4}$ Escuela de Tecnología Médica, Facultad de Ciencias, Universidad Mayor, Temuco, Chile. \\ *Autor para correspondencia: andrea.fontenele@yahoo.com.br \\ RECIBIDO: 23 de mayo 2021 \\ APROBADO: 19 de julio 2021
}

DOI: $10.22370 /$ bolmicol.2021.36.1.2924

Palabras claves: Transplante Renal; Micose; Antifúngicos; Farmácia Clínica, Aspergillus, Candida, Cryptococcus.

Keywords: Kidney Transplantation; Mycosis; Antifungal Agents; Clinical Pharmacy.

\section{RESUMEN}

O transplante renal é uma alternativa eficaz como tratamento da Doença Renal Crônica, dado seu custo efetividade e o aumento na sobrevida dos pacientes. Os imunossupressores também garantem maior sobrevida do enxerto, porém tornam o receptor susceptível a infecções, como as fúngicas. O objetivo do estudo foi conhecer as infecções fúngicas de pacientes da enfermaria de transplante renal do Hospital de Referência do Maranhão, através de pesquisa dos prontuários destes com diagnóstico de micose entre 2014 a 2018. Foram encontrados 9 casos, 5 de criptococose, 2 de aspergilose e 2 de candidíase, sendo 6 pacientes homems e 3 mulheres; 5 receptores de doadores vivos e 4, doadores falecidos. Em 7 casos, a terapia de imunossupressão prescrita foi metilprednisolona e basiliximab, e em 2 casos, timoglobulina. $\mathrm{O}$ exame diagnóstico foi o micológico direto em 6 casos e endoscopia digestiva alta, biópsia de pele e exame clínico em 1 caso cada. As espécies isoladas foram Cryptococcus sp. (3), C. neoformans (2), Aspergillus sp. (2), Candida tropicalis, C. krusei (1) e C. albicans (1). Excetuando-se um caso de candidíase tratado com fluconazol e um caso com a forma desoxicolato, todos os outros foram tratados com anfotericina B lipídica e fluconazol como manutenção. Sete casos tiveram a cura como desfecho, e 2 o óbito. Percebe-se a importância do estudo dessas infecções, o olhar cuidadoso em relação as mesmas e a atuação do farmacêutico para monitorização da farmacoterapia, para maior segurança do paciente. 
Perfil das infecções fúngicas em pacientes transplantados renais - Fontenele A. M. et al.

\section{ABSTRACT}

Renal transplantation has been an effective alternative as the treatment of Chronic Renal Disease, due to its cost effectiveness and the increase of the patients' survival. Immunosuppressants also ensure the longer survival of the graft, but make the recipient susceptible to infections, such as fungi. The objective of the study was to know as the main fungal infections of patients with transplant renal disease at the Reference Hospital of Maranhão, through the study of the medical records of patients diagnosed with fungal infection in the period from 2014 to 2018 . There were 9 cases, cryptococcosis, 2 aspergillosis and 2 per candidiasis, 6 male and 3 female patients, 5 live donor recipients and 4 deceased donors. In 7 cases, a prescribed immunosuppressive therapy was methylprednisolone and basiliximab, and in 2 cases thymoglobulin. The clinical examination was done in 6 cases and upper digestive endoscopy, skin biopsy and clinical examination in 1 case each. The species were Cryptococcus sp. (3), C. neoformans (2), Aspergillus sp. (2), Candida tropicalis, $C$. krusei (1) and C. albicans (1). Except for one case of candidacy treated with fluconazole and one case with a deoxycholate form, all others were treated with amphotericin B lipid complex and fluconazole as maintenance. Seven cases had a cure as an outcome, and 2 a death. It was noticed the importance of the study of these infections, the careful look at them and the pharmacist's performance to monitor pharmacotherapy, for greater patient safety.

\section{INTRODUCCIÓN}

A Doença Renal Crônica (DRC) no estágio V apresenta a hemodiálise (HD), diálise peritoneal (DP) e o transplante renal (TxR) como formas de tratamento substitutivo. O transplante renal é uma das principais formas de tratamento da medicina moderna na doença renal crônica em estágios mais avançados, promovendo qualidade de vida e aumento da sobrevida desses pacientes. $1,2,3$

Nos últimos 30 anos, com as tecnologias da imunossupressão (ciclosporina, tacrolimo, azatioprina e micofenolato) contribuem para uma preservação eficaz dos órgãos e, mesmo com os altos custos iniciais, os gastos pósprocedimento são diminuídos após 2,6 anos. $\mathrm{O}$ transplante renal é considerado a melhor alternativa dentre as terapias renais substitutivas (TRS), e inclusive mais custo efetiva que a hemodiálise a partir do segundo ano do transplante, sendo que esta última modalidade garante maior expectativa e qualidade de vida. ${ }^{1,4}$

O Brasil detém um vasto programa de transplantes de órgãos, que em sua maioria são custeados pelo Sistema Único de Saúde (SUS), através do Sistema Nacional de Transplantes. O SUS possui estabelecimentos de saúde e equipes médicas habilitadas em 25 estados da federação. No Maranhão, hospitais de referência como o Hospital da Universidade Federal do Maranhão (HUUFMA), foram determinantes para o desenvolvimento dos centros de transplante em todo o estado. Estudos recentes realizados na Central de Transplantes do HUUFMA indicam alta sobrevida em 1- 3 e 5 anos pós-transplante, sendo o doador vivo ou falecido, e taxas de óbitos consonantes a parâmetros nacionais e internacionais preconizados na literatura ${ }^{3,5}$.

Para realização do transplante renal é previamente necessária a dessensibilização do paciente, de modo que haja uma menor probabilidade de rejeição, fazendo uso contínuo de medicamentos imunossupressores. A imunossupressão, traz consigo a possibilidade de instauração de quadros de infecções oportunistas, dentre eles as infecções fúngicas. ${ }^{6}$

A alta taxa de mortalidade de pacientes acometidos com infecções fúngicas, bem como a perda da função do enxerto, deve-se principalmente à dificuldade e demora do diagnóstico clínico e laboratorial, retardando o início do tratamento antifúngico. ${ }^{7}$ Dessa forma, 
Perfil das infecções fúngicas em pacientes transplantados renais - Fontenele A. M. et al.

tem-se a terapia medicamentosa como o principal manejo das infecções fúngicas, e o farmacêutico, profissional especialista neste insumo, contribui para promover uma terapia adequada e o uso racional dos medicamentos, diminuição das reações adversas e dos custos do tratamento, Através do acompanhamento farmacoterapêutico do recém transplantado, quer seja na enfermaria ou no ambulatório o farmacêutico contribui também para a adesão medicamentosa, diminuindo as recidivas da doença. ${ }^{8}$

\section{MATERIALES Y MÉTODOS}

Foi realizado um estudo observacional, retrospectivo e prospectivo, descritivo, com abordagem quantitativa, obtido a partir de um estudo de prevalência de infecções fúngicas em transplantados renais no período de janeiro de 2014 a novembro de 2018 (número CAAE 96417318.6.0000.5086). A pesquisa foi realizada na Unidade de Transplante do Hospital da Universidade Federal do Maranhão Foram incluídos todos os pacientes transplantados renais acompanhados na enfermaria do transplante renal do HUUFMA, que apresentaram diagnóstico de infecção fúngica, de ambos os sexos e todas as faixas etárias. Foram excluídos do estudo pacientes que deram entrada na enfermaria do transplante renal com quadro de outras infecções ou outros motivos de internação que não infecções fúngicas. A pesquisa foi feita mediante consulta dos prontuários dos pacientes, não sendo necessário preenchimento de Termo de Consentimento
Livre e Esclarecido (TCLE). Os dados coletados foram armazenados e exibidos em forma de gráficos, tabelas e quadros por meio do Microsoft Office 2013.

\section{RESULTADOS}

Foram encontrados nove casos de infecções fúngicas, sendo cinco por Cryptococcus, dois por Aspergillus e dois por Candida (Tabla 1). A média de idade foi de cinquenta e um anos, sendo seis pacientes do sexo masculino e três do sexo feminino. Dentre os achados, cinco foram receptores de doadores vivos e quatro de doadores falecidos. Em sete dos casos, a terapia de imunossupressão prescrita foi metilprednisolona e basiliximab (MP+BSX), e em dois pacientes, timoglobulina (Thymo). O exame diagnóstico foi o micológico direto em seis achados, e endoscopia digestiva alta (EDA), biópsia de pele e exame clínico em um caso cada. Os fungos isolados foram Cryptococcus spp. (3 isolamentos), C. neoformans (2 achados), Aspergillus spp. (1) A. fumigatus (1), Candida tropicalis e C. krusei (1) e C. albicans (1). Excetuando-se um caso de candidíase, tratado com fluconazol e um caso com a forma desoxicolato, todos os outros foram tratados com anfotericina B complexo lipídico como escolha para tratamento e fluconazol como manutenção, salvo um dos pacientes com aspergilose, onde optou-se pelo tratamento com itraconazol. Sete indivíduos obtiveram a cura como desfecho clínico, e dois o óbito.

\section{Tabla 1: Casos de infecção fúngica em transplantados renais entre os anos de 2014 e 2018 , no HUUFMA, Maranhão, Brasil, 2019.}

\begin{tabular}{ccccccccc}
\hline Nome & Sexo & Idade & Data do TxR & $\begin{array}{c}\text { ISS } \\
\text { (indução) }\end{array}$ & ISS (manutenção) & $\begin{array}{c}\text { Tipo de } \\
\text { doador }\end{array}$ & $\begin{array}{c}\text { Exame } \\
\text { diagnóstico }\end{array}$ & Amostra \\
\hline 1 & M & 68 & $06 / 07 / 2014$ & MP+BSX & $\begin{array}{c}\text { FK/PRED/ } \\
\text { MPS }\end{array}$ & Falecido & $\begin{array}{c}\text { Micológico } \\
\text { direto }\end{array}$ & Urina \\
\hline 2 & M & 50 & $06 / 07 / 2014$ & MP+BSX & $\begin{array}{c}\text { FK/PRED/ } \\
\text { MPS }\end{array}$ & Vivo & $\begin{array}{c}\text { Micológico } \\
\text { direto }\end{array}$ & Líquor \\
\hline
\end{tabular}


Perfil das infecções fúngicas em pacientes transplantados renais - Fontenele A. M. et al.

\begin{tabular}{|c|c|c|c|c|c|c|c|c|}
\hline 3 & M & 57 & $18 / 05 / 2014$ & $\mathrm{MP}+\mathrm{BSX}$ & $\begin{array}{c}\text { FK/PRED/ } \\
\text { MPS }\end{array}$ & Falecido & $\begin{array}{c}\text { Micológico } \\
\text { direto }\end{array}$ & $\begin{array}{l}\text { Lav. } \\
\text { Brônquico }\end{array}$ \\
\hline 4 & $\mathrm{~F}$ & 61 & 29/10/2011 & Thymo & $\begin{array}{c}\text { FK/PRED/ } \\
\text { MPS }\end{array}$ & Vivo & $\begin{array}{c}\text { Micológico } \\
\text { direto }\end{array}$ & Líquor \\
\hline 5 & $\mathrm{~F}$ & 35 & $16 / 12 / 2004$ & $\mathrm{MP}+\mathrm{BSX}$ & $\begin{array}{c}\text { MMF/PRED/ } \\
\text { SRL }\end{array}$ & Vivo & Exame clínico & Líquor \\
\hline 6 & $\mathrm{~F}$ & 58 & $20 / 04 / 2015$ & Thymo & $\begin{array}{c}\text { FK/PRED/ } \\
\text { MMF }\end{array}$ & Vivo & Biópsia & Pele \\
\hline 7 & M & 44 & 25/06/2009 & $\mathrm{MP}+\mathrm{BSX}$ & $\begin{array}{c}\text { FK/PRED/ } \\
\text { MPS }\end{array}$ & Vivo & $\begin{array}{l}\text { Micológico } \\
\text { direto }\end{array}$ & $\begin{array}{l}\text { Líquido } \\
\text { pleural }\end{array}$ \\
\hline 8 & M & 61 & $02 / 06 / 2015$ & $\mathrm{MP}+\mathrm{BSX}$ & $\begin{array}{c}\text { FK/PRED/ } \\
\text { MPS }\end{array}$ & Falecido & $\begin{array}{c}\text { Micológico } \\
\text { direto }\end{array}$ & $\begin{array}{l}\text { Líquido de } \\
\text { perfusão }\end{array}$ \\
\hline 9 & M & 71 & $09 / 02 / 2018$ & $\mathrm{MP}+\mathrm{BSX}$ & FK/PRED/MPS & Falecido & EDA & Fragmento \\
\hline
\end{tabular}

Tabla 2: Casos de infecção fúngica em transplantados renais entre os anos de 2014 e 2018 , no HUUFMA, Maranhão, Brasil, 2019 (continuación).

\begin{tabular}{|c|c|c|c|c|c|c|c|}
\hline Nome & $\begin{array}{c}\text { Data do } \\
\text { diagnóstico }\end{array}$ & $\begin{array}{c}\text { Intervalo } \\
\text { TxR/ } \\
\text { diagnóstico } \\
\text { (dias) }\end{array}$ & Fungo isolado & $\begin{array}{c}\text { Data início } \\
\text { de } \\
\text { tratamento }\end{array}$ & $\begin{array}{c}\text { Terapia } \\
\text { anti } \\
\text { fúngica }\end{array}$ & $\begin{array}{l}\text { Tempo de } \\
\text { tratamento } \\
\quad \text { (dias) }\end{array}$ & Desfecho \\
\hline 1 & $18 / 08 / 2014$ & 43 & C. neoformans & $18 / 08 / 2014$ & $\begin{array}{c}\text { ABCL+ } \\
\text { FCZ }\end{array}$ & 195 & CURA \\
\hline 2 & $14 / 05 / 2014$ & 39 & $\begin{array}{c}\text { Cryptococcus } \\
\text { spp }\end{array}$ & $15 / 08 / 2014$ & $\mathrm{ABCL}+$ & 337 & CURA \\
\hline 3 & 03/06/2014 & 16 & $\begin{array}{c}\text { Cryptococcus } \\
\text { spp }\end{array}$ & $04 / 06 / 2014$ & $\mathrm{ABD}$ & 14 & OBITO \\
\hline 4 & $22 / 03 / 2016$ & 1606 & C. neoformans & $29 / 03 / 2016$ & $\begin{array}{c}\text { ABCL+ } \\
\text { FCZ }\end{array}$ & 365 & CURA \\
\hline 5 & $05 / 12 / 2017$ & 4737 & Não isolado & $05 / 12 / 2017$ & ABCL+ & $\begin{array}{c}\text { Em } \\
\text { tratamento }\end{array}$ & CURA \\
\hline 6 & $10 / 11 / 2015$ & 204 & Aspergillus spp. & $10 / 11 / 2015$ & $\begin{array}{c}\text { ABCL+ } \\
\text { FCZ }\end{array}$ & 29 & ÓBITO \\
\hline 7 & $25 / 04 / 2018$ & 3226 & $\begin{array}{c}\text { Aspergillus } \\
\text { fumigatus }\end{array}$ & $25 / 04 / 2018$ & $\begin{array}{c}\text { ABCL+ } \\
\text { ICZ }\end{array}$ & 134 & CURA \\
\hline 8 & 03/06/2015 & 1 & $\begin{array}{c}\text { C.tropicalis e } \\
\text { C.krusei }\end{array}$ & $17 / 06 / 2015$ & ABCL & 9 & CURA \\
\hline 9 & $04 / 04 / 2018$ & 54 & Candida spp. & $05 / 04 / 2018$ & FCZ & 7 & CURA \\
\hline
\end{tabular}

Leyenda: ABCL (anfotericina B complexo lipídico); FCZ (fluconazol); ABD (anfotericina B desoxicolato); ICZ (itraconazol). HUUFMA: Hospital universitário, Universidade Federal de Maranhão.

\section{DISCUSIÓN}

As infecções fúngicas, são de baixa incidência em pacientes transplantados renais, mas apresenta alta mortalidade conforme a literatura.
Dentre vários fatores, pode-se destacar a inespecificidade dos sintomas, demora no diagnóstico e rápida evolução da doença, acrescido do perfil imunossuprimido dos pacientes transplantados. As infecções mais 
Perfil das infecções fúngicas em pacientes transplantados renais - Fontenele A. M. et al.

recorrentes descritas são: aspergilose, candidíase e criptococose. Estão associadas mais frequentemente a pacientes recémtransplantados, relacionados a fatores como falência renal, uso de concentrado de hemácias, além do tratamento imunossupressor. ${ }^{9,14}$

\section{Aspergilose}

A aspergilose, infecção fúngica mais recorrente, producida por fungos filamentosos que se reproduzem por conídios. Apresenta-se geralmente na forma pulmonar invasiva em imunodeprimidos, podendo ser precoce (nos primeiros seis meses pós TxR) ou mais tardia. A espécie mais comum nestes casos é o $A$. fumigatus, que se apresenta microscopicamente pelos conídios com a cabeça aspergilar, e em colônias de coloração azul esverdeada quando semeada em placa de agar malte. Recomenda-se o rápido início do tratamento quando se há suspeita deste diagnóstico, devido evolução acelerada do estado do paciente. Os dois pacientes com este fungo, notou-se um caso atípico de aspergilose cutânea e outro característico de aspergilose pulmonar invasiva (API). Somente em um dos pacientes (caso 7) a espécie foi identificada, como sendo o $A$. fumigatus, mais comumente associada a esta manifestação da doença. ${ }^{10,11}$

\section{Candidíase}

A candidíase é comumente relacionada a pacientes imunossuprimidos nos seis primeiros meses de transplante, sendo que a literatura mostra como espécies mais incidentes a $C$. albicans, $C$. tropicalis, $C$. krusei e $C$. glabrata. Os três primeiros se apresentam como leveduras de brotamento com formato elíptico, apesar de serem capazes, quando no tecido infectado, de exibir também morfologia filamentosa. A $C$. albicans e $C$. tropicalis tem a capacidade de formar tanto hifas verdadeiras quanto pseudohifas, já a espécie C. krusei apenas forma pseudo-hifas; essas três espécies tipicamente são maiores que a $C$. glabrata. $^{12,13}$
Os sítios mais frequentes de infecção são: trato gastrintestinal (TGI), sistema respiratório e trato urinário. Nos dois casos apresentados, há a presença de dois destes patógenos, além de outra espécie, C. krusei, menos incidente, mas também relacionado a infecções em pacientes imunossuprimidos, além de doenças hematológicas, com características específicas como multirresistência e resistência intrínseca a fluconazol. Os sítios de infecção apresentados confluem com o que foi acima descrito (líquido de perfusão renal e TGI). ${ }^{13}$

\section{Criptococose}

A criptococose caracteriza-se por uma infecção fúngica sistêmica causada por espécies do gênero Cryptococcus, sendo o Cryptococcus neoformans e o Cryptococcus gatti as espécies de relevância clínica. Essas espécies são basidiomicetos que se apresentam, no tecido do hospedeiro, como leveduras encapsuladas de aspecto refringente. Comumente, subaguda a crônica, a micose se apresenta de duas formas: criptococose oportunista, em populações imunodeprimidas, sendo o $C$. neoformans a espécie predominante desses casos; e a criptococose primária, atribuída ao $C$. gattii que atinge, com maior frequência, os imunocompetentes das regiões endêmicas, que são as zonas tropicais e subtropicais. ${ }^{14,15}$

Nos casos encontrados, esta doença possui maior incidência, indo de encontro à literatura, que aponta a aspergilose como micose sistêmica mais frequente. Não somente a grande presença de aves, outrossim de substrato vegetal característico das regiões Norte e Nordeste, como o Sygygium jambolana (jambolão), Eucalyptus camaldulensis (eucalipto) e Theobroma cacao (cacau), contribuem para discussão da alta incidência de criptococose, pois a literatura aponta que o fungo pode ser encontrado nessas espécies de árvores. ${ }^{16}$

A espécie C. neoformans apresentou dois isolamentos, com outros dois achados onde se definiu apenas o gênero (Cryptococcus spp.) e 
Perfil das infecções fúngicas em pacientes transplantados renais - Fontenele A. M. et al.

um diagnóstico unicamente clínico (caso 5). Apesar de a literatura indicar a candidíase e aspergilose como infecções fúngicas mais comuns, é válido salientar a predominância de casos de criptococose (cinco) em relação às outras micoses sistêmicas, evidenciando o perfil de patógenos que acometeram os receptores de transplante renal do hospital. ${ }^{11}$

\section{Perfil das infecções fúngicas}

Os imunossupressores de indução prescritos em sete dos nove casos foram MP+BSX, e dois pacientes fizeram uso de THYMO, devido ao alto risco imunológico, conforme o Kidney Diseases: Improving Global Outcomes (KDIGO), que recomenda a terapia imunossupressora de indução com uso de um agente biológico numa primeira instância (BSX), ou de um agente redutor de linfócitos (THYMO), este último em casos de receptores com alto risco imunológico. ${ }^{17}$

Também se percebe uma maior incidência de infecção em receptores de doadores vivos que de doadores falecidos nesta amostra. Um estudo de revisão sistemática e meta-análise indica maior incidência de infecções nesta segunda população em relação a primeira, o que leva a questionamentos acerca do agente da infecção, característica da infecção fúngica, adesão ao tratamento imunossupressor, ou ainda polimorfismo genético. ${ }^{6}$

Em seis casos, o diagnóstico ficou definido através de exame micológico direto, amplamente utilizado na prática clínica pelo seu baixo custo e rapidez na realização. Concomitantemente, realiza-se a cultura para fungos, com diagnóstico um pouco mais tardio, mas que possibilita a identificação da espécie fúngica responsável. Os outros exames foram utilizados em casos mais específicos, conforme decisão da equipe médica e tecido infectado. ${ }^{18}$

Os protocolos utilizados para o tratamento das infecções fúngicas são descritos em bases de dados de alto nível de evidência, como o
UpToDate e o Micromedex. Os cinco casos de criptococose foram tratados com ABCL $5 \mathrm{mg} / \mathrm{kg} /$ dia IV por duas semanas na fase inicial, seguindo com o uso de fluconazol $200 \mathrm{mg}$ a $400 \mathrm{mg} /$ dia VO como terapia de manutenção, de seis a doze meses. Apenas um dos achados (caso 3) foi tratado com ABD. Nos casos de aspergilose, os tratamentos foram ABCL $5 \mathrm{mg} / \mathrm{kg}$ IV durante seis semanas, interrompido pelo óbito da paciente (caso 6), e ABCL $5 \mathrm{mg} / \mathrm{kg}$ IV durante duas semanas como fase de indução, e itraconazol 200mg VO por dezesseis semanas como fase de manutenção. Já nos casos de candidíase, um deles (caso 8) foi tratado com ABCL $5 \mathrm{mg} / \mathrm{kg}$ IV durante dez dias, e o outro (caso 9) com fluconazol 200mg IV por quatorze dias. ${ }^{19}$

\section{Atuação do farmacêutico nas infecções fúngicas}

Integrado a equipe multiprofissional, o farmacêutico se responsabiliza pela farmacoterapia do paciente. O tratamento das micoses sistêmicas apresentadas em sete dos nove casos foi feito com ABCL, cuja aquisição foi feita mediante preenchimento de formulário correspondente e encaminhado ao Ministério da Saúde através do Setor de Farmácia do Hospital. Esse medicamento possui ainda outras duas formas: desoxicolato e lipossomal. A primeira, apesar de disponível, não foi utilizada devido a sua nefrotoxicidade aguda; a segunda, mesmo sendo eficaz nesses casos, é somente liberada pelo Programa de Leishmaniose. A liberação da ABCL restringe-se aos casos de micoses sistêmicas endêmicas e algumas oportunistas em pacientes não portadores HIV. ${ }^{20}$

Dentro da farmacoterapia do transplante renal, detectam-se problemas relacionados a medicamentos, sendo a interação medicamentosa um dos mais frequentes. A terapia imunossupressora, necessária para manutenção do enxerto renal, interage com os medicamentos antifúngicos de forma farmacocinética, uma vez que competem pelo substrato CYP3A4 do citocromo P450, onde há 
Perfil das infecções fúngicas em pacientes transplantados renais - Fontenele A. M. et al.

deslocamento do inibidor de calcineurina (tacrolimo), aumentando a sua biodisponibilidade e levando a toxicidade do mesmo e aumento significativo do risco de complicações cardíacas, como arritmia e fibrilação ventricular. ${ }^{20,21}$

Nesses casos, a intervenção farmacêutica e crucial para avaliar o aprazamento mais adequado entre os fármacos, a fim de diminuir a possibilidade de interação, bem como sugerir a equipe médica possíveis mudanças na terapia, como diminuição ou mesmo suspensão do tratamento imunossupressor por um período. Deve-se avaliar sempre o custo benefício, dado o alto risco de mortalidade das infecções fúngicas. A presença do profissional farmacêutico sugere, a partir de evidência científica, o aprazamento da terapia baseado no tempo de infusão da droga, preconizando tempo superior a quatro horas antes da administração do inibidor de calcineurina, minimizando os efeitos adversos. ${ }^{21}$

A partir dos dados apresentados, percebe-se que as infecções causadas por fungos são de baixa incidência, mas de alta mortalidade. Ademais, há discordâncias entre os casos apresentados e os encontrados na literatura, a saber: infecção fúngica mais recorrente, tipos de doadores e taxas de óbitos, mostrando o perfil da flora fúngica do hospital, com as suas peculiaridades, bem como a necessidade de mais estudos e pesquisas a fim de elucidar este assunto, como o projeto do qual este artigo foi criado se propõe. Neste contexto clínico, o profissional farmacêutico é importante para uma condução adequada da farmacoterapia em todos os aspectos, proporcionando o uso racional e favorecendo o sucesso de transplante e a segurança do paciente.

\section{AGRADECIMIENTOS}

Agradecemos à equipe de transplante renal do Hospital Universitário, pelo auxílio na coleta de dados dos prontuários, a equipe de laboratório pela disponibilidade de dados dos diagnósticos, à Universidade Federal do Maranhão - UFMA e pela Residência Multiprofissional do Hospital Universitário da Universidade Federal do Maranhão - HUUFMA, pelo fomento e apoia a esta pesquisa.

Conflicto de interés: los autores no tienen conflictos de interés.

\section{REFERENCIAS}

1. Garcia, G; Harde, P; Chapman, J. et al. The global role of kidney transplantation. Kidney and Blood Pressure Research. 2012;(35): p.299-304.

2. Bommer, J. Prevalence and socio-economic aspects of chronic kidney disease. Nephrology Dialysis Transplantation. 2002; 17 (11): 8-12.

3. Guimaraes, Thaíse Almeida; Rodrigues, Wanderson Barros; Fontenele, Andréa Martins Melo. Infecções fúngicas em transplantados renais: uma revisão integrativa. Revista de Pesquisa em Saúde. 2017: 119-23.

4. Silva, S; Caulliraux, H; Silva,C; Rocha,E. Uma comparação dos custos do transplante renal em relação às diálises no Brasil. Cad. Saúde Pública, Rio de Janeiro.2016, 32(6): e00013515.

5. Oliveira, Maria Inês Gomes de; Santos, Alcione Miranda dos; Salgado Filho, Natalino. Survival analysis and associated factors to mortality of renal transplant recipients in a University Hospital in Maranhão. J Bras Nefrol. 2012; 34(3):216-25.

6. Taminato, M; $\quad$ Fram,D; Grothe,C;Rodrigues,R; Belasco,A; Barbosa,D. Prevalência de infecção em transplante renal de doador vivo versus falecido: revisão sistemática e metanálise. Esc Enferm USP. 2015; 49(3):509-14.

7. Badiee, P; Alborzi, A. Invasive fungal infections in renal transplant recipients. Exp Clin Transplant.2011: 355-62. 
Perfil das infecções fúngicas em pacientes transplantados renais - Fontenele A. M. et al.

8. Calabró, A.A; Follador, W. Custoefetividade de voriconazol versus anfotericina B no tratamento da aspergilose invasiva. RBM. 2003; 60(7): $528-35$.

9. Quindós, G. Candidiasis, aspergilosis y otras micosis invasoras en receptores de trasplantes de órgano sólido. Rev Iberoam Micol. 2011; 28(3)11019 .

10. Monteiro, F; Rato da Conceição M. Caracterização de isolados de Aspergillus provenientes de ambiente hospitalar - identificação molecular e determinação dos padrões de susceptibilidade aos antifúngicos. 2017. Dissertação (Mestrado em Biologia Humana e Ambiente) Universidade de Lisboa, Lisboa, 2017.

11. Pérez-Sáez, Maria José et al. Invasive Aspergillosis in Kidney Transplant Recipients: A Cohort Study. Experimental and Clinical Transplantation. 2014; 02: 101-05, 31.

12. Schell, W.A; Kauffman C.A; Thorner, A.R. Biology of Candida infections. Disponível em: https://www.uptodate.com/contents/biology-ofcandida-

infections?search=genero $\% 20$ candida\&source $=$ sear ch_result\&selectedTitle= $1 \sim 150 \&$ usage_type $=$ default $\&$ display_rank $=1$. Acesso em: 30 jan 2019.

13. Pfaller, M. A. Candida krusei, a MultidrugResistant Opportunistic Fungal Pathogen: Geographic and Temporal Trends from the ARTEMIS DISK Antifungal Surveillance Program, 2001 to 2005. J. M C, 2008; 46:

14. Cox, G.M; Perfect, JR. Microbiology and epidemiology of Cryptococcus neoformans infection. UptoDate®, 2016. Disponível em: http://www.uptodate.com/contents/microbiologyand-epidemiology-of-cryptococcus-neoformansinfection Data de acesso: 02 ago 2018.

15. Cox, G.M; Perfect, JR. Cryptococcal infection outside the central nervous system. UptoDate ${ }^{\circledR}$ 2017. Disponível em: http://www.uptodate.com/contents/cryptococcus- neoformans-infection-outside-the-central-nervoussystem. Data de acesso: 02 ago 2018.

16. BRASIL. Ministério da Saúde. Vigilância epidemiológica da criptococose. Brasília, abril de 2012.

17. Brennan, D.C; Daller, J.A; Lake, KD. Pharm, C.D; Castillo, D. for the Thymoglobulin Induction Study Group. Rabbit Antithymocyte Globulin versus Basiliximab in Renal Transplantation. N Engl J Med, 2006; 355; 19:196777.

18. Souza, P.R.M. Concordância entre o exame micológico direto e cultural em infecções ungueais dos pés por dermatófitos. Dissertação (Mestrado em Ciências Médicas) - U Federal do Rio Grande do Sul, Porto Alegre, 2007.

19. MICROMEDEX. Amphotericin B Liquid Complex - Adult Dosing. Disponível em: https://www-micromedexsolutions com.ez14.periodicos.capes.gov.br/micromedex2/libr arian/PFDefaultActionId/evidencexpert.DoIntegrate dSearch?navitem $=$ topHome \&is ToolPage=true\# .

Acesso em: 30 jan 2019.

\section{AGÊNCIA NACIONAL DE VIGILÂNCIA} SANITÁRIA (ANVISA). Consulta a banco de dados. Medicamentos. Disponível em http://portal.anvisa.gov.br. Acesso em 29 de jan 2019.

21. Fuchs, F.D; Wannmacher, L. Farmacologia Clínica e Terapêutica. Ed. Guanabara Koogan, 2017. 